\title{
A heuristic model inversion for coupled thermo-hydro-mechanical modeling of triaxial experiments
}

\author{
Jack Lin ${ }^{1}$, Mustafa Sari ${ }^{1}$, Manolis Veveakis ${ }^{2}$, Thomas Poulet ${ }^{3,1, *}$
}

\begin{abstract}
As multiphysics geomechanical models get developed, their increasing complexity and number of parameters make it particularly difficult to calibrate against experimental data. In this contribution, we present a heuristic workflow to invert for parameters of a coupled Thermo-Hydro-Mechanical (THM) model in a way that helps the theoretical modellers refine their definition of the underlying elasto-visco-plastic model itself. We apply this workflow to the calibration of deviatoric and volumetric data for two sets of triaxial experiments on mudstone and sandstone. We show that beyond the calibration of well-defined parameters of the THM model, one or two scaling factors of the experimentally obtained yield surface and two more factors to capture the confinement and pore pressure dependency of the flow law lead to the satisfactory matching of series of experiments at varying confinements for two different rock types. Using this physical model, we also show that tracking the volumetric component of the mechanical power in the numerical simulations might allow reducing the number of experiments required to calibrate the model.
\end{abstract}

Keywords: Coupled THM elasto-visco-plasticity, Parameter inversion, Sensitivity analysis

\footnotetext{
*thomas.poulet@csiro.au

${ }^{1}$ School of Minerals and Energy Resources Engineering, UNSW Sydney, NSW 2052, Australia

${ }^{2}$ Civil and Environmental Engineering, Duke University, NC 27708, USA

${ }^{3}$ CSIRO Mineral Resources, Kensington, WA 6151, Australia
} 


\section{Introduction}

Geomechanics laboratories worldwide perform triaxial experiments on a daily basis, for the purpose of understanding rock behaviours and measuring relevant properties used by diverse theoretical models in academia and various industries. Given the long history of geomechanics, comprehensive experimental results and observations exist nowadays to characterise most rocks around ambient conditions, also providing a fair insight into the corresponding sensitivities with respect to temperature, pore pressure, confinement, or loading rate, just to name a few. The most conventional manner to account for such dependencies has consisted for a long time in approaching geomechanical modelling from a purely mechanical perspective, using traditional constitutive models, and account for the variations of other (secondary) physical processes like temperature and pressure through their influence on the parameters used in the constitutive model (e.g. yield envelope). While successfully providing an empirical way to incorporate more physics in geomechanics, this approach still presents some limitations to tackle situations where feedback couplings between physical processes are too strong, as is the case for the study of material instabilities for example. For those applications, tighter couplings are sought, motivated by the fundamental understanding of the physical processes at play from a multiphysics perspective. The non-mechanical processes are then considered at the same level at the mechanics itself, acknowledging that non-noticeable temperature or pressure changes at the sample scale, during a triaxial experiment, do not prevent temperature or pressure changes at the micro-scale from affecting the overall rock behaviour and localisation in particular, as was shown for mudstone for example (Poulet and Veveakis, 2016). Considering the relative infancy and increased complexity of those mutliphysics models, however, begs the question of calibrating the input parameters required to match experimental results.

Inverting for numerical parameters of a theoretical model to match experimental results can be challenging, even for a simple model with a low number of parameters, because of the overall uncertainty arising from cumulative errors at all levels: theoretical, experimental and numerical (see Table 1).

Uncertainty is probably most intuitively accepted at the experimental level, where physical limitations of the experimental apparatus (precision based on quality), including the measuring equipment, are obvious factors. 
They are, however, not the only ones. Rocks themselves are naturally inhomogeneous and it is difficult to obtain enough representative samples, as the determination of a Representative Elementary Volume (REV) is a difficult task in itself. Human factors must also be taken into account, regarding the choice and quality of the sampling (accidental damage, contamination), calibration of the machines and other possible mishandling mistakes. Uncertainty at the numerical level is also obvious as rounding errors and risks of hardware failure are well understood. Yet, those effects are probably negligible compared to the risks arising from programmers and operators when selecting the theoretical model, implementing the numerical tools, defining the meshing level, determining the input parameter design (for stochastic generation) or simply inputting data. Uncertainty also arises at the theoretical level itself, due to the need of listing of physical processes considered (under- or over-estimation) and all interactions (known or unknown) between those physical processes, which affect the size of the RVE. Modellers can also select unsuitable theoretical models, not necessarily by lack of understanding of all hypotheses behind the models, but also because other physical processes than mechanics can affect those applicability hypotheses in a manner that is simply not yet fully understood. Dealing with this uncertainty led to the active development of inversion methods based on multi-objective optimisation methods (see (Nguyen-Tuan et al., 2016) and citations within), but no single perfect method has been identified yet as there is "no free lunch" (Wolpert and Macready, 1997). Every benefit from a given approach comes with less interesting consequences, and this inversion problem becomes exponentially more difficult as the number of variables increases, leading to an intractable problem so-far for most multiphysics approaches.

Metaheursitics are specifically developed to tackle these inversion problems using mathematical optimisation and computer science techniques, dealing with the uncertainties at the experimental and numerical levels with as little as possible human interaction for scalability purposes. Sometimes, however, operators cannot be completely removed from the process, which needs to be interactive (Wijns et al., 2004). This is the case in particular if one wants to account for uncertainties at the theoretical level and modify the constitutive model used during the optimisation process itself (see Fig. 1). This applies in particular for theoretical modellers aiming at developing new multiphysics models and is the focus of this study.

In this contribution we present a model inversion for coupled ThermoHydro-Mechanical (THM) modeling of triaxial experiments, which is heuris- 
Table 1: Non-exhaustive list of sources of uncertainties

\begin{tabular}{|l|l|l|l|}
\hline Types & $\begin{array}{l}\text { Sources of uncer- } \\
\text { tainty }\end{array}$ & Human factors & Non-human factors \\
\hline \multirow{2}{*}{ Theoretical } & $\begin{array}{l}\text { Physical processes con- } \\
\text { sidered } \\
\text { Constitutive models }\end{array}$ & $\begin{array}{l}\text { Under/over-estimation of pro- } \\
\text { cesses at play } \\
\text { Applicability }\end{array}$ & $\begin{array}{l}\text { Complex feedbacks between pro- } \\
\text { cesses }\end{array}$ \\
\hline \multirow{2}{*}{ Experimental } & Material sampling & $\begin{array}{l}\text { Sampling choices, sampling qual- } \\
\text { ity (damage, contamination) }\end{array}$ & Natural representativity \\
& Experiment apparatus & Calibration, mishandling & Machine quality, precision \\
\cline { 2 - 5 } & Measuring equipment & Calibration, mishandling & Machine quality, precision \\
\hline \multirow{2}{*}{ Numerical } & simulation setup & $\begin{array}{l}\text { Evaluation of theoretical model, } \\
\text { mesh coarseness, input parameter } \\
\text { design, input errors }\end{array}$ & - \\
& $\begin{array}{l}\text { Imperfect/incomplete code verifi- } \\
\text { cation (implementation errors) }\end{array}$ & rounding errors, hardware failure \\
& Ing libraries & Implementation & rounding errors, hardware failure \\
\hline
\end{tabular}

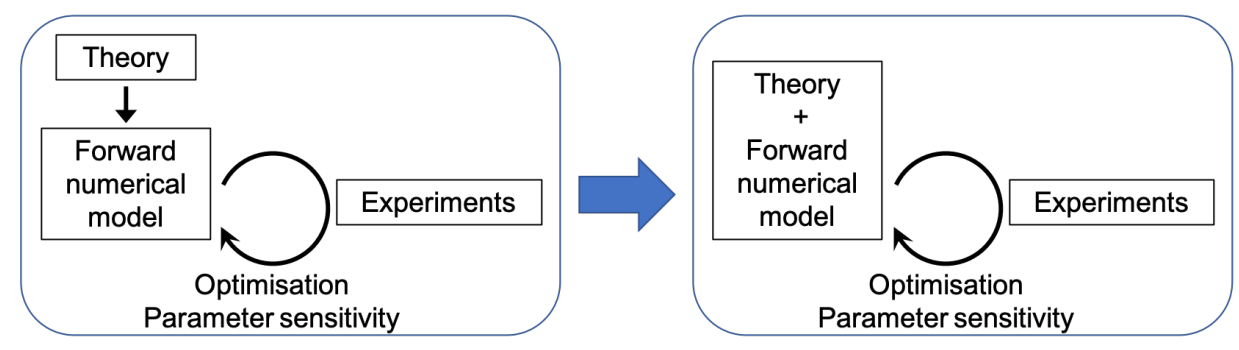

Figure 1: Schematic workflows (A) most commonly and (B) in this study.

tic in the literal definition of the term, i.e. enabling the theoretical modellers to learn from the optimisation worklow to refine their multiphysics model. The aim is to extend the work of (Poulet and Veveakis, 2016) and describe the process for capturing the temperature and pressure dependencies of the mechanical enthalpy formulation on which the constitutive model is based. Section 2 describes the multiphysics geomechanical model used. Section 3 presents the inversion workflow to calibrate the model against experiments and Section 4 the application of that workflow to two sets of experiments on mudstone and sandstone. Finally, Section 5 discusses the interest of this approach, facilitating the development of the underlying theoretical model.

\section{Uncertainties of the geomechanical model}

The theoretical model at the core if this study is an elasto-visco-plastic model that was specifically developed to investigate material instabilities 
like pore collapse (Poulet and Veveakis, 2016). The model is based on the traditional momentum, mass and energy conservation equations of a mixture (denoted by the subscript $m$ ) composed of a solid matrix (subscript $s$ ) fully saturated with fluid (subscript $f$ ), along with constitutive equations described in detail in (Poulet and Veveakis, 2016). Neglecting gravity and any chemical reaction and advection terms in the context of quasi-static triaxial experiments, these equations express the evolution of the system as

$$
\begin{aligned}
0 & =\partial_{j} \sigma_{i j}^{\prime}-\partial_{i} p_{f} \\
0 & =\beta_{m} \partial_{t} p_{f}-\partial_{i}\left[\frac{\kappa}{\mu_{f}} \partial_{i} p_{f}\right]-\lambda_{m} \partial_{t} T+v_{i, i}^{(s)}, \\
0 & =\left(\rho C_{p}\right)_{m} \frac{D T}{D t}-\partial_{i i} T-\chi \sigma_{i j} \dot{\epsilon}_{i j}^{p l}
\end{aligned}
$$

where $\sigma^{\prime}$ represents the effective stress, $\beta$ the compressibility, $\lambda$ the thermal expansion coefficient, $\kappa$ the permeability, $\mu$ the viscosity, $v^{(s)}$ the solid velocity, $\rho$ the density, $C_{p}$ the specific heat, $\chi$ the Taylor-Quinney coefficient, and $\dot{\epsilon}^{p l}$ the plastic strain rate.

Following (Poulet and Veveakis, 2016), we introduce the following normalised variables

$$
\begin{aligned}
p^{*} & =\frac{p_{f}}{\sigma_{r e f}}, \\
T^{*} & =\frac{T-T_{r e f}}{T_{r e f}}, \\
x^{*} & =\frac{x}{x_{r e f}}, \\
t^{*} & =\frac{c_{t h, r e f}}{x_{r e f}^{2}} t,
\end{aligned}
$$

where $\sigma_{r e f}, T_{r e f}, x_{r e f}$ and $c_{t h, r e f}$ are reference values for the stress, temperature, length and thermal diffusivity of the mixture respectively. Using all normalised variables and dropping the asterisk notation for simplicity, we work in this study with the following system of equations expressing the evolution of the temperature $(T)$, pore fluid pressure $\left(p_{f}\right)$ and displacement 
$\left(u_{i}, 1 \leq i \leq 3\right)$ as

$$
\begin{aligned}
& 0=\partial_{j} \sigma_{i j}^{\prime}-\partial_{i} p_{f} \\
& 0=\partial_{t} p_{f}-\partial_{i}\left[\frac{1}{L e} \partial_{i} p_{f}\right]-\Lambda \partial_{t} T+\frac{P e \dot{\epsilon_{V}}}{\beta_{m}}, \\
& 0=\partial_{t} T-\partial_{i i} T-G r \sigma_{i j} \dot{\epsilon}_{i j}^{p l}
\end{aligned}
$$

This formulation uses dimensionless groups including the Lewis number Le, Gruntfest number $G r$ and thermal pressurisation $\Lambda$ defined as

$$
\begin{aligned}
& L e=\frac{\mu_{f} c_{t h, r e f} \beta_{m}}{\kappa} \\
& G r=\frac{\sigma_{r e f}}{T_{r e f}\left(\rho C_{p}\right)_{m}} \chi \\
& \Lambda=\frac{\lambda_{m} \delta T_{r e f}}{\beta_{m} \sigma_{r e f}} .
\end{aligned}
$$

The main particularity of this model, built on the overstress plasticity framework of Perzyna (1966) and proposed by Poulet and Veveakis (2016), is that dependencies on state variables are expressed through the definition of the mechanical enthalpy rather than more traditional expressions like the yield envelope and hardening modulus. The intent is to capture the temperature and pressure dependencies of the constitutive model with a physical description (see Sari, 2019), which is a worthy goal but also introduces more uncertainty in terms of model calibration compared to more engineering approaches. For instance, the evolution of the hardening modulus is not an input of the model, as is the case for many models where it is defined as a function of (for example) strain, and in which it can be therefore be monitored rather accurately in the lab and entered directly in simulation to reproduce numerically those laboratory results. Instead, the hardening or softening behaviour of our model can only be altered by changing the values of material parameters who will in turn impact the stress response, including through variations of temperature or pore pressure, or by modifying the plastic flow law itself (Poulet and Veveakis, 2016) through the definition of the mechanical enthalpy $Q_{m e c h}$, or its normalised Arrhenius variable $A r=\frac{Q_{m e c h}}{R \tilde{T}_{0}}$, with $\tilde{T}_{0}$ the temperature in Kelvin. The flow law used reads

$$
\dot{\epsilon}_{i j}^{v p}=\dot{\epsilon}_{0} \bar{\sigma}^{m} \exp ^{\frac{A r T}{1+T}} \frac{\partial f}{\partial \sigma_{i j}}
$$


where $\epsilon^{v p}$ denotes the visco-plastic stress, $\epsilon_{0}$ a pre-exponential factor, $\bar{\sigma}$ the overstress, $m$ a material parameter, $f$ the plastic flow potential and $R$ the gas constant.

In this approach, particular attention is paid to the temperature and pore pressure evolutions, which might appear puzzling at first sight when modelling experiments on rocks under isothermal conditions.

\subsection{Temperature effect}

Most triaxial tests are performed under isothermal conditions (often room temperature), which would make it a fair assumption to neglect the temperature effects when modelling the corresponding experiments. The temperature, however, does vary internally due to all dissipative processes taking place at the micro-scale and the temperature increase becomes clearly evident with infrared radiation (IRR) imaging when a fracture occurs (Wu et al., 2006). While such changes can be accurately monitored with IRR cameras at the precise (and short-lived) moment when a rock sample breaks, which even allows to monitor temperature as a precursor for rock fracturing and failure (Wu et al., 2006), the external temperature monitoring of a triaxial experiment will unfortunately reveal no evolution until localisation is well pronounced. Indeed, most themal imaging cameras are usually sensitive to gradations of 0.05-0.15 degress Celcius, which is not precise enough to detect micro heat signature events, especially if not located on the outside of the sample. The temperature itself, though, is undeniably increasing through dissipation and its effects on the yield stress for instance are well captured by Eyring plots (e.g. Poulet and Veveakis, 2016). We are therefore considering temperature as a primary variable in our framework, where its evolution is mainly constrained through the values of the Gruntfest number $(G r)$. Its effect on the mechanics is controlled by the Arrhenius number $(A r)$.

\subsection{Pore pressure effect}

Similarly to temperature, pore fluid pressure can also play a considerable role at the microstructural level, even in cases where it's overall value at the sample level seems rather constant. The normalised mass balance equation (3b) shows the impact of the Lewis number $(L e)$, expressing the ratio of thermal over mass diffusivities. It accounts therefore not only for the preponderant effect of fluid permeability, but also for any other internal mass diffusion process, including those resulting from physical processes occurring at the micro-scale like pore collapse (Sari, 2019). As such, we also track pore 
pressure as primary variable, whose value is strongly affected by the Lewis number $L e$, thermal pressurisation coefficient $\Lambda$ and compressibility $\bar{\beta}$. Its variation affects the effective stress directly, by definition, and less directly through the pressure dependency of the mechanical dissipation. Those effects impact, in turn, on the temperature through the mechanical dissipation.

\subsection{Rate sensitivity}

The rate sensitivity of geomaterials is another well accepted phenomenon (e.g. Poulet and Veveakis, 2016), which plays an important role when modelling rocks at geological time scales. While considerable efforts are being spent in measuring flow laws from experiments for as many materials as possible under various conditions, as well as developing theoretical models to capture those dependencies (e.g. Mielke, 2006), no consensus exists yet and the problem remains an active research area. Unsurprisingly, the difficulty lies in capturing simultaneously the various cross-dependencies between temperature, pressure and rate. For instance, recent observations showed the increased rate-dependency of calcarenite with compression (Sari, 2019).

\subsection{Plasticity model}

Those sensitivities to temperature, pressure and rate can be captured at various levels in the geomechanical model, which contains itself some inherent uncertainty as many of its constituents are being investigated in the quest for an improved modelling approach.

\subsubsection{Activation enthalpy}

The main characteristic of the geomechanical approach we're following is the explicit formulation of the mechanical enthalpy $Q_{\text {mech }}$. While the formula initially proposed (Poulet and Veveakis, 2016) remains a guideline, its exact definition is the subject of ongoing research (Sari, 2019) and needs therefore to be considered itself as a free set of parameters in the overall inversion approach of experimental results. Theoretical considerations can provide some constraints on the enthalpy definition (Sari, 2019), but no definitive form has been universally accepted yet. In this contribution, we account therefore for the related uncertainty by taking the definition (Poulet and Veveakis, 2016)

$$
Q_{m e c h}=\alpha_{1}+\alpha_{2} p_{f}
$$


where $\alpha_{1}$ and $\alpha_{2}$ are material parameters to be inverted for specific experiments.

\subsubsection{Yield envelope}

Another distinctive aspect of our mechanical approach is the definition of the yield surface itself, which differs from the one taken by most traditional geomechanics models. The only available data regarding the yield surface obviously comes from experimental curves, which exhibit characteristic points that can be interpreted as yield points. Both on stress-strain curves from triaxial experiments and isotropic compression tests, an initial straight line is usually interpreted as a linear elastic response, which allows the identification of a yield point as the point on the curve where an arbitrarily determined deviation from that linear elastic response occurs (see Fig. 2). It is important to note the two major problems with this determination method, on top of the obvious uncertainty stemming from the experimental results themselves.

Firstly, a yield point can only be determined, by definition, using data from unloading experiments. The initial linear trend of experimental curves does indeed not always correspond to an elastic behaviour, as was shown for the case of calcarenite for instance (Sari, 2019). In that example, even the first unloading sequences during this initial (loading) linear response showed that the rock had reached plasticity from the very beginning. Such complex responses raise questions about the existence of a yield envelope altogether.

Secondly, experimental data provide information for a whole sample, which is supposed to be representative. The numerical simulations done to calibrate any geomechanical model, however, use a discretised mesh composed of smaller cells on which the material behaviour is computed and there is no reason, in general, for any of those smaller cells to be a REV. Experimental data provide therefore precious information about yield and failure at the sample scale, whereas the yield envelope needed to simulate numerically the same experiment is at a lower scale. The difference in scales implies that the yield envelope needed for the simulations can only be smaller than the experimentally obtained one, and in this work we consider the two to differ only by a scaling function, which is taken into account as extra parameters to invert for.

In this contribution, we use a capped model which is well adaptd to the two case studies presented below (see Sec. 4). Specifically, we work with an enhanced version of the modified Cam-Clay model (Gerolymatou, 2017) 

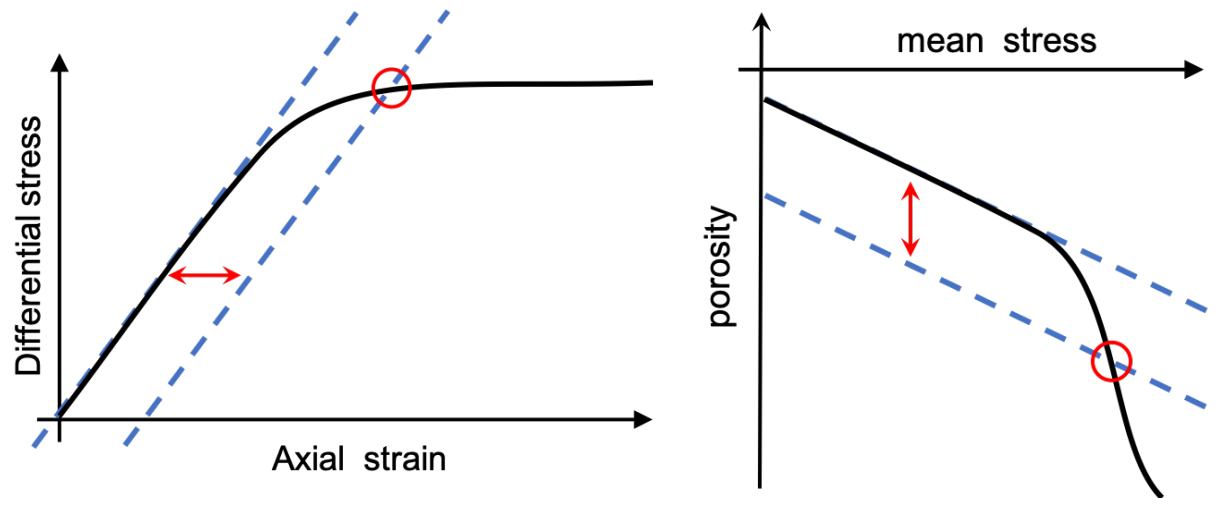

Figure 2: Schematic representation of two main methods to determine a yield point (circled) from (a) a triaxial experiment or (b) an isotropic compression test, using an exaggerated offset (distance between parallel dashed lines) for illustration purposes.

which proposes a yield surface defined as

$$
q^{2}+M^{2} h(p)\left(p-p_{t}\right)\left(p-p_{c}\right)=0
$$

where $p$ denotes the mean effective stress, $q$ the deviatoric stress, $M$ the slope of the critical state line, $p_{c}$ the preconsolidation pressure and $p_{t}$ the tension cut-off. A dependence on mean pressure is expressed through the function

$$
h(p)=\exp \left[-\frac{1}{\gamma_{1}}\left(\frac{p-p_{t}}{p_{c}-p_{t}}-\gamma_{2}\right)^{2}\right]
$$

where $\gamma_{1}$ and $\gamma_{2}$ are numerical parameters affecting the shape and skewness of the yield surface. Note that this yield surface can lose convexity for certain values of the parameters and needs to be used with care. This definition provides a more flexible yield envelope than the Modified Cam-Clay model, with the ability to break the symmetry by shifting the top point of the envelope.

To constrain the determination of the yield envelope and minimise the number of free parameters, a first envelope is determined by matching the reported experimental data, providing values for all parameters $M, p_{c}, p_{t}, \gamma_{1}$ and $\gamma_{2}$.

We allow ourselves a maximum of two degrees of freedom to identify the best yield envelope as a transformation of the experimental curve, determined 
by a new pre-consolidation pressure $\tilde{p}_{c}$ and new maximum value of deviatoric stress $\tilde{q}_{\max }$, scaling the initial envelope along the Critical State Line joining the tension cut-off with the peak of the envelope of coordinates $\left(p_{\max }, q_{\max }\right)$ in $(p-q)$ space. The corresponding value of the mean effective stress $\tilde{p}_{\max }$ is therefore defined as

$$
\tilde{p}_{\max }=p_{t}+\frac{\tilde{q}_{\max }}{q_{\max }}\left(p_{\max }-p_{t}\right)
$$

A scaled version of the envelope can then be obtained with modified values $\tilde{\gamma}_{2}$ and $\tilde{M}$ defined as

$$
\begin{gathered}
\tilde{\gamma}_{2}=2\left(\frac{\tilde{p}_{\max }-p_{t}}{\tilde{p}_{c}-p_{t}}-\gamma_{1}\right) \frac{\left(\tilde{p}_{\max }-p_{t}\right)\left(\tilde{p}_{c}-\tilde{p}_{\max }\right)}{\left(\tilde{p}_{c}-p_{t}\right)\left(\tilde{p}_{c}+p_{t}-2 \tilde{p}_{\max }\right)} \\
\tilde{M}=\tilde{q}_{\max } \exp \left[\frac{1}{2 \tilde{\gamma}_{2}}\left(\frac{\tilde{p}_{\max }-p_{t}}{\tilde{p}_{c}-p_{t}}-\gamma_{1}\right)^{2}\right] \frac{1}{\sqrt{\left(\tilde{p}_{\max }-p_{t}\right)\left(\tilde{p}_{c}-\tilde{p}_{\max }\right)}}
\end{gathered}
$$

The result section (Sec. 4) presents two application examples, illustrated in Figs. 5 and 8. Note that stresses are represented as positive in compression on all figures.

\section{Inversion workflow}

In this contribution we focus on the inversion workflow required to identify all parameters needed to match numerical simulations of confined drained triaxial experiments with experimental data. The numerical setup is defined in details in (Poulet and Veveakis, 2016) and simulates a standard confined drained experiment with constant temperature and no flow imposed as boundary conditions on the sides of the 3D block. As the longer term goal is to investigate the underlying multiphysical processes involved, we consider all samples to be homogeneous and attribute a single value for each of the material parameters, rather than try to improve the match by introducing spatial distributions for different samples. Considering the "no-free-lunch-theorem" (Wolpert and Macready, 1997), no existing inversion strategy performs better than all others. Based on that, we have hereby developed a customised approach for the problem at hand. 


\subsection{Model parameters}

From all considerations presented in Sec. 2, a list of parameters to be inverted for can be established (see Table. 3.1), including the material parameters but also the constitutive models parameters. In particular, as mentioned above, we allow ourselves a maximum of two free parameters to rescale the yield envelope, and we also invert for the two parameters $\alpha_{1}$ and $\alpha_{2}$ of the mechanical enthalpy. Most parameters represent usual material properties and can be constrained in reasonably tight ranges from literature and experimental data. A few parameters, including the Lewis number Le, Gruntfest number $G r$, and mechanical enthalpy parameters $\alpha_{1}$ and $\alpha_{2}$ are more poorly constrained.

\begin{tabular}{|c|c|c|}
\hline Parameter name & Symbol & $\begin{array}{l}\text { Unit of } \\
\text { Measure }\end{array}$ \\
\hline Flow law overstress exponent & $M$ & - \\
\hline Initial permeability & $\kappa$ & $m^{2}$ \\
\hline Initial porosity & $\phi$ & - \\
\hline Arrhenius coefficient & $A r$ & - \\
\hline Young's modulus & $E$ & $\mathrm{~Pa}$ \\
\hline Poisson ratio & $\nu$ & - \\
\hline Fluid thermal diffusivity & $c_{t h, f}$ & $m^{2} \cdot s^{-1}$ \\
\hline $\begin{array}{l}\text { Fluid thermal expansion coeffi- } \\
\text { cient }\end{array}$ & $\lambda_{f}$ & $K^{-1}$ \\
\hline Fluid viscosity & $\mu, f$ & Pa.s \\
\hline Fluid compressibility & $\beta_{f}$ & $P a^{-1}$ \\
\hline Solid thermal diffusivity & $c_{t h, s}$ & $m^{2} \cdot s^{-1}$ \\
\hline Soild thermal expansion coefficient & $\lambda_{s}$ & $K^{-1}$ \\
\hline Solid compressibility & $\beta_{m}$ & $P a^{-1}$ \\
\hline Mixture thermal diffusivity & $c_{t h, m}$ & $m^{2} \cdot s^{-1}$ \\
\hline $\begin{array}{l}\text { Mixture thermal expansion coeffi- } \\
\text { cient }\end{array}$ & $\lambda_{s}$ & $K^{-1}$ \\
\hline Mixture compressibility & $\beta_{m}$ & $P a^{-1}$ \\
\hline $\begin{array}{l}\text { Mixture thermal pressurisation co- } \\
\text { efficient }\end{array}$ & $\Lambda_{m}$ & - \\
\hline Lewis number & Le & - \\
\hline Gruntfest number & $G r$ & - \\
\hline
\end{tabular}




\begin{tabular}{|l|l|l|} 
Yield envelope, preconsolidation & $\tilde{p}_{c}$ & $\mathrm{~Pa}$ \\
pressure & $\tilde{q}_{\max }$ & $\mathrm{Pa}$ \\
$\begin{array}{l}\text { Yield envelope, maximum devia- } \\
\text { toric stress }\end{array}$ & $\alpha_{1}$ & - \\
\hline Mechanical enthalpy parameter $\alpha_{1}$ & $\alpha_{2}$ & - \\
\hline Mechanical enthalpy parameter $\alpha_{2}$ & $\alpha_{2}$ &
\end{tabular}

Table 2: List of all parameters to invert for in the numerical simulations of triaxial experiments

\subsection{Optimisation algorithm}

Numerous meta-heuristic approaches have been previously developed to invert model parameters from experimental data in geomechanics, and following (Nguyen-Tuan et al., 2016) we are using a Particle Swarm Optimisation (PSO) computational paradigm to perform this task, as it is particularly well suited to investigate optimisation problems with multiple local minima as faced in a mutliphysics context. We use an implementation of PSO algorithm that was specifically developed and integrated with our computational framework (Lin et al., 2017) to run on the various computational facilities available to us.

In order to reduce the computational time and maximise the learning benefit from the optimisation process, not all parameters are inverted for automatically but the process is broken down in several stages.

The inversion process starts by selecting numerical values for all parameters, including reference values for normalisation, as best estimates based on reported values from the experiment, literature values for the rock involved, and previous studies. This provides a starting point from which the parameter optimisation can proceed, following three steps (illustrated in Fig. 3). The first step consists in using PathFinder (Lin et al., 2017) to optimise the numerical values of $\alpha_{1}$ and $\alpha_{2}$ for each of the confinement cases individually. As a second step, the scaling parameter of the yield envelope is then optimised manually. The third step sees the adjustment of all other variables, manually or using PathFinder. In practise, this involves mainly the three variables identified as playing the most important roles: the thermal pressurisation coefficient $\Lambda$, Gruntfest number $(G r)$ and Lewis number $(L e)$. These three steps are repeated iteratively until satisfactory convergence is obtained. 


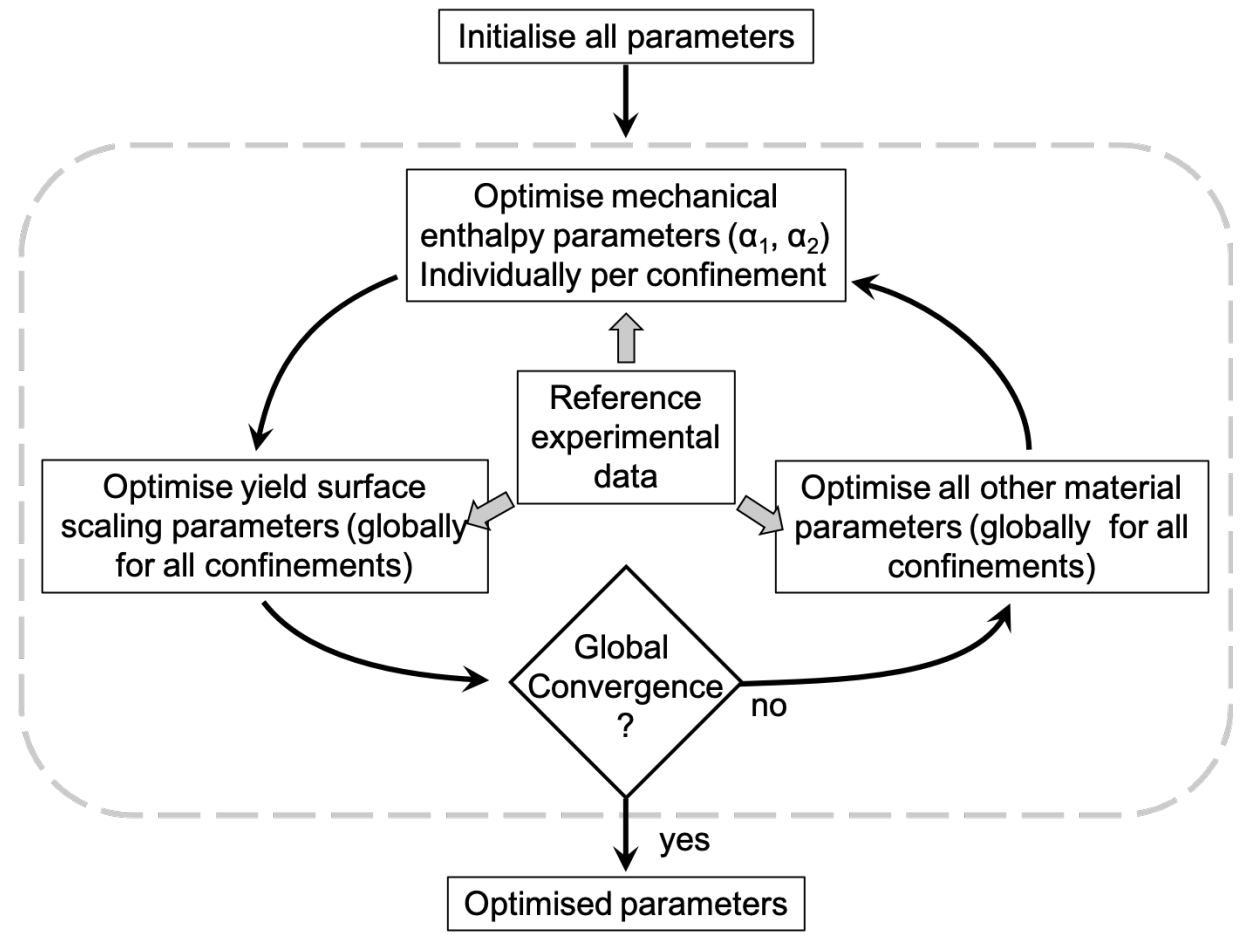

Figure 3: Schematic description of the optimisation process, broken down in three main steps to optimise sequentially the flow law parameters, yield surface scaling parameters and all other material parameters until global convergence is reached. 


\subsection{Objective function}

Given a series of $N$ confined drained experiments $C D_{i}$ with $1 \leq i \leq N$ at various confinement pressures, an automatic parameter inversion can be performed through a minimisation of an objective function $f$, which is taking into account both the volumetric and deviatoric components of the experimental and simulation data to reflect the importance of those two components in the model formulation (see Poulet and Veveakis, 2016). See Fig. 4 for a graphical representation of the computation. For each experiment $i$, the deviatoric component $f_{i}^{d}$ of the objective function is expressed as the L2-norm of the difference between the experimental and numerical simulation results of the strain-stress curve for that experiment. Similarly, the volumetric component $f_{i}^{d}$ represents the L2-norm of the error on the porosity evolution as a function of strain. To increase the focus on the impact of the constitutive model in plasticity, both definitions are taken on a subset of the whole range of strain values, from a manually selected value of strain after all experiments display a plastic behaviour until the end of the experiments (horizontal range in between dashed vertical lines on Fig. 4). In other words, we are more interested in the plastic behaviour at large strain than on the elastic regime or its transition to the plastic regime. The overall deviatoric and volumetric objective functions, $f^{d}$ and $f^{v}$, are taken as the sums of all components for all confining pressures, $f^{d}=\sum_{i=1}^{N} \omega_{i} f_{i}^{d}$ and $f^{v}=\sum_{i=1}^{N} \omega_{i} f_{i}^{v}$, using a family of weights $\left\{\omega_{i}\right\}_{1 \leq i \leq N}$ which typically reduces the emphasis on the low values of confinement where the physical model is less adapted. In order to respect some balance between the volumetric and deviatoric components, the values of stress and porosity used in the definition of the errors are normalised so their maximum ranges are identical. The total objective function is taken as the sum of the deviatoric and volumetric components, $f=f_{d}+f_{v}$.

\section{Results}

The inversion workflow presented in Sec. 3 was used to calibrate the model parameters (see Sec. 3.1) for two sets of experimental data containing six experiments each. In both cases, the weights values of the objective function were selected as $\{0.2,0.5,1,1,1,1\}$ to reduce the impact of the experiments at the lower confinements. 


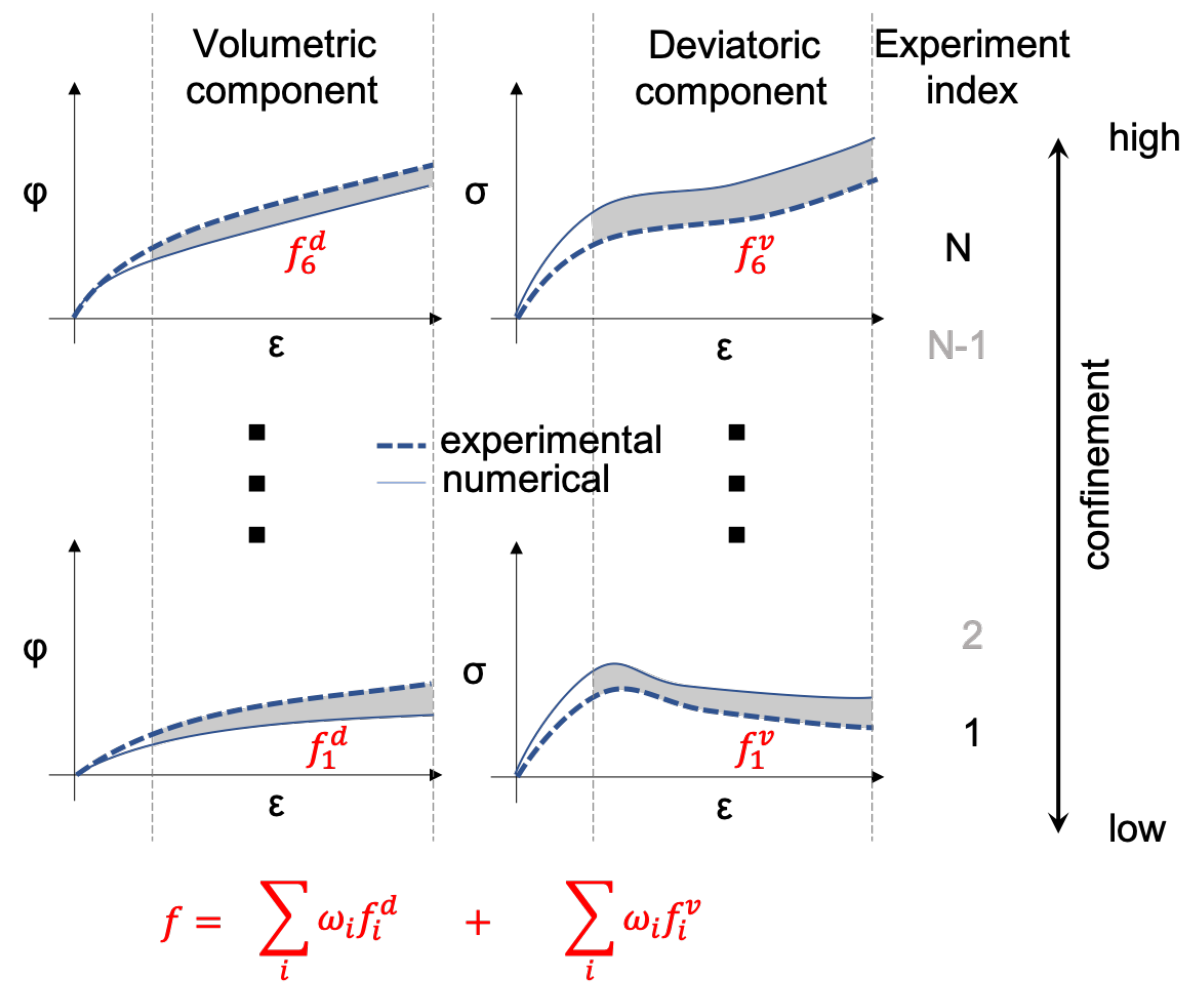

Figure 4: Schematic description of the objective function $(f)$ computation, showing its volumetric and deviatoric components for a series of experiments at various confinements. See the full explanation in Sec. 3.3. 


\subsection{Diatomaceous mudstone}

The first case study focuses on reported confined drained triaxial experiments performed by Oka et al. (2011) on Noto diatomaceous mudstone, a rock composed of $1 \%$ sand, $66 \%$ silt and $33 \%$ clay and characterised by its high porosity $(73 \%)$ and permeability $\left(1.55 \times 10^{-9} \mathrm{~m}^{2}\right)$. Six confined drained tests, named CD1-6, were conducted at effective confinement pressures of $0.25,0.5,0.75,1,1.5$ and $2 \mathrm{MPa}$ respectively, on fully water saturated rectangular prisms samples of $4 \times 4 \times 8 \mathrm{~cm}$, with the longitudinal direction of all samples taken perpendicular to the plane of sedimentation. All experiments started with an initial equilibration step of up to 6 hours to reach the desired confinement, followed by a $20 \%$ strain compression operated at constant displacement of $0.1 \% / \mathrm{min}$ (see Oka et al., 2011, for details).

The strain-stress results from those experiments were previously used to calibrate a previous version of the model (Poulet and Veveakis, 2016). In this work, we consider in addition the volumetric responses as functions of mean effective stress, as extra simultaneous constraints.

The experimental results from (Oka et al., 2011) indicate the suitability of the extended modified Cam-Clay yield envelope (see Section. 2.4.2) for the whole sample. A first set of numerical values is derived to match the experimental yield points reported by Oka et al. (2011): $M=1.2, p_{c}=$ 2.26MPA, $p_{t}=-0.68 \mathrm{MPa}, \gamma_{1}=0$ and $\gamma_{2}=1 e 6$. Note that the large value of $\gamma_{2}$ basically produced a traditional Modified Cam-Clay envelope. Based on the discussion from Sec. 2.4.2 we are conjecturing a similar but smaller shape for the yield envelope needed for the numerical simulations, keeping only a single free scaling parameter since keeping the same preconsolidation pressure ultimately proved adequate.

Following the inversion process described in Section 3.2 leads to the final parameters listed in table 4.1, providing acceptable fits for both sets of curves shown in Fig. 6 and Fig. 7.

\begin{tabular}{|l|l|l|l|}
\hline Parameter name & Symbol & Value & $\begin{array}{l}\text { Unit of } \\
\text { Measure }\end{array}$ \\
\hline Flow law overstress exponent & $M$ & 2 & - \\
\hline Initial permeability & $\kappa$ & $\begin{array}{l}1.55 \\
10^{-9}\end{array}$ & \\
& & $m^{2}$ \\
\hline Initial porosity & $\phi$ & 0.74 & - \\
\hline Arrhenius coefficient & $A r$ & 8 & - \\
\hline
\end{tabular}




\begin{tabular}{|c|c|c|c|}
\hline Young's modulus & $E$ & 135.6 & $\mathrm{MPa}$ \\
\hline Poisson ratio & $\nu$ & 0.2 & - \\
\hline Fluid thermal diffusivity & $c_{t h, f}$ & $\begin{array}{l}1.43 \times \\
10^{-7}\end{array}$ & $m^{2} \cdot s^{-1}$ \\
\hline $\begin{array}{l}\text { Fluid thermal expansion coeffi- } \\
\text { cient }\end{array}$ & $\lambda_{f}$ & $6.9 \times 10^{-5}$ & $K^{-1}$ \\
\hline Fluid viscosity & $\mu, f$ & $8.9 \times 10^{-4}$ & Pa.s \\
\hline Fluid compressibility & $\beta_{f}$ & $\begin{array}{l}4.5 \\
10^{-10}\end{array}$ & $P a^{-1}$ \\
\hline Solid thermal diffusivity & $c_{t h, s}$ & $\begin{array}{l}1.64 \times \\
10^{-6} \quad \times\end{array}$ & $m^{2} \cdot s^{-1}$ \\
\hline Solid thermal expansion coefficient & $\lambda_{s}$ & $5 \times 10^{-6}$ & $K^{-1}$ \\
\hline Solid compressibility & $\beta_{m}$ & $\begin{array}{l}3.46 \quad \times \\
10^{-7}\end{array}$ & $P a^{-1}$ \\
\hline Mixture thermal diffusivity & $c_{t h, m}$ & $\begin{array}{l}5.45 \quad \times \\
10^{-7}\end{array}$ & $m^{2} \cdot s^{-1}$ \\
\hline $\begin{array}{l}\text { Mixture thermal expansion coeffi- } \\
\text { cient }\end{array}$ & $\lambda_{s}$ & $\begin{array}{l}5.18 \\
10^{-5}\end{array}$ & $K^{-1}$ \\
\hline Mixture compressibility & $\beta_{m}$ & $\begin{array}{l}9.34 \quad \times \\
10^{-8}\end{array}$ & $P a^{-1}$ \\
\hline $\begin{array}{l}\text { Mixture thermal pressurisation co- } \\
\text { efficient }\end{array}$ & $\Lambda_{m}$ & 555 & Pa.K \\
\hline Lewis number & Le & 50 & - \\
\hline Gruntfest number & $G r$ & $3.3 \times 10^{-3}$ & - \\
\hline Yield envelope, tension cut-off & $p_{t}$ & -0.68 & $\mathrm{MPa}$ \\
\hline $\begin{array}{l}\text { Yield envelope, preconsolidation } \\
\text { pressure }\end{array}$ & $p_{c}$ & 2.26 & $\mathrm{MPa}$ \\
\hline $\begin{array}{l}\text { Yield envelope, slope of critical } \\
\text { state line }\end{array}$ & $M$ & 0.24 & - \\
\hline Yield envelope, $\gamma_{1}$ parameter & $\gamma_{1}$ & 10 & - \\
\hline Yield envelope, $\gamma_{2}$ parameter & $\gamma_{2}$ & -31.4 & - \\
\hline
\end{tabular}

Table 3: Parameter values obtained for the fits of mudstone experiments shown in Fig. 6 and Fig. 7. 


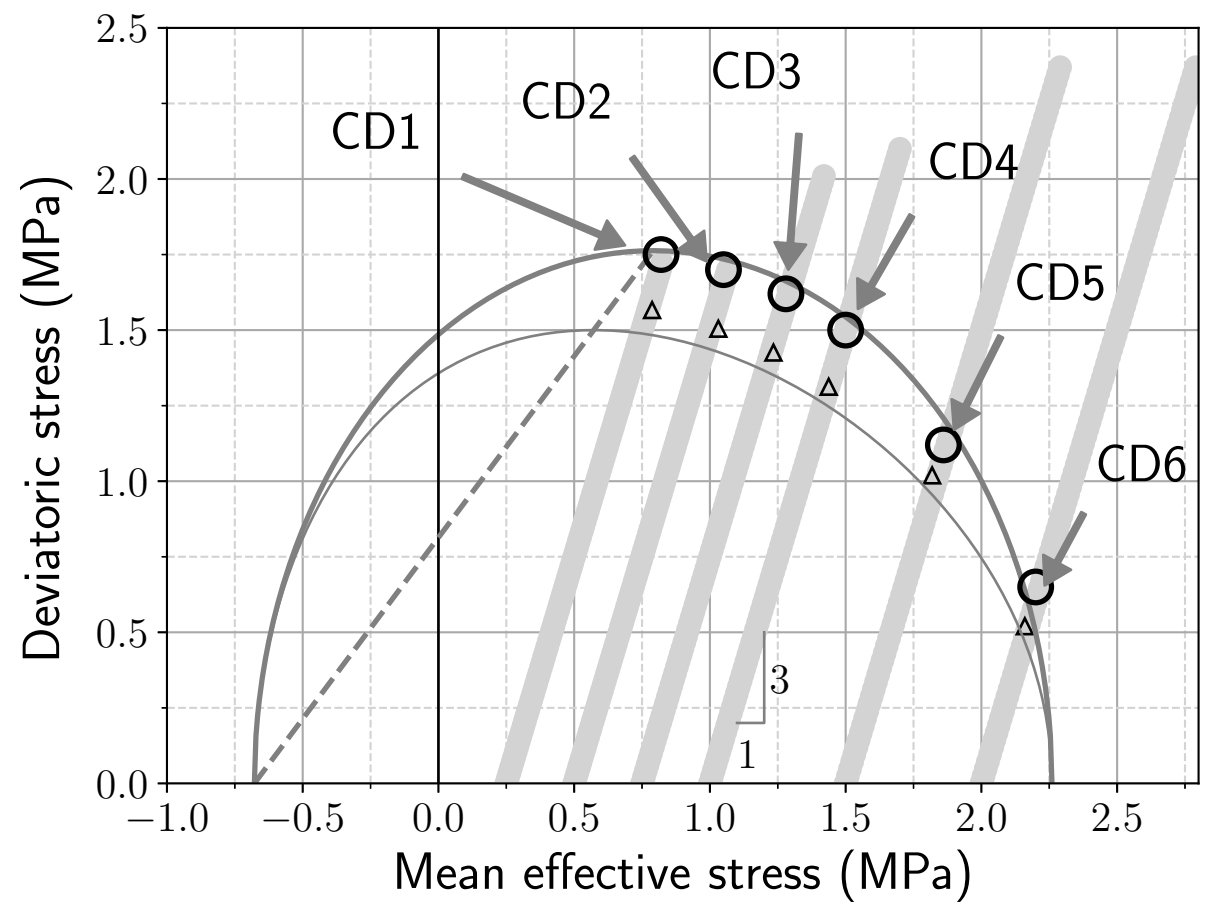

Figure 5: Experimentally determined yield points (black circles) for mudstone by Oka et al. (2011) and corresponding yield envelope (thin line), as well as result yield envelope (thick line) obtained from the inversion process, scaling the experimental yield envelope along the Critical State Line (dashed line). The thick lines in light grey show the stress paths for all confined drained experiments CD1-6. The triangles mark yield points obtained from the experimental data at $0.2 \%$ plastic strain. 


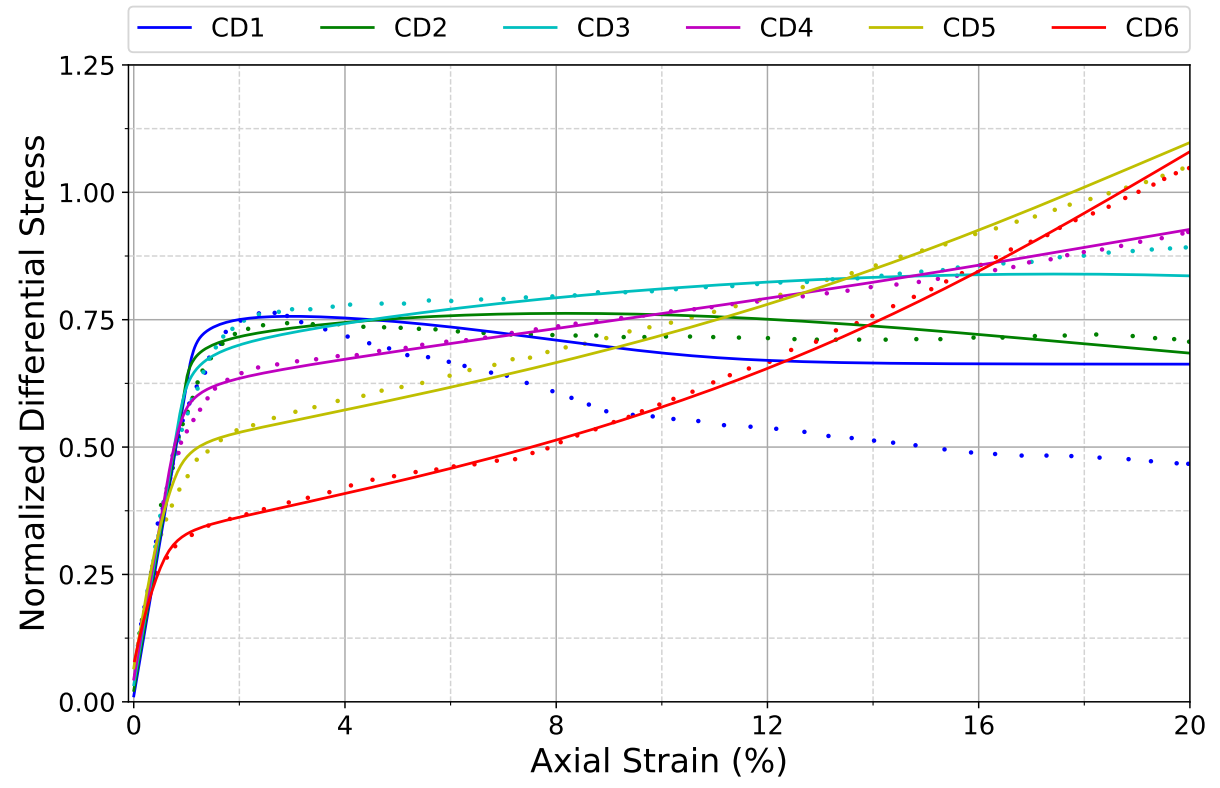

Figure 6: Stress-strain curves at various confinement for mudstone; comparison of numerical (solid lines) and experimental results (dashed lines). 


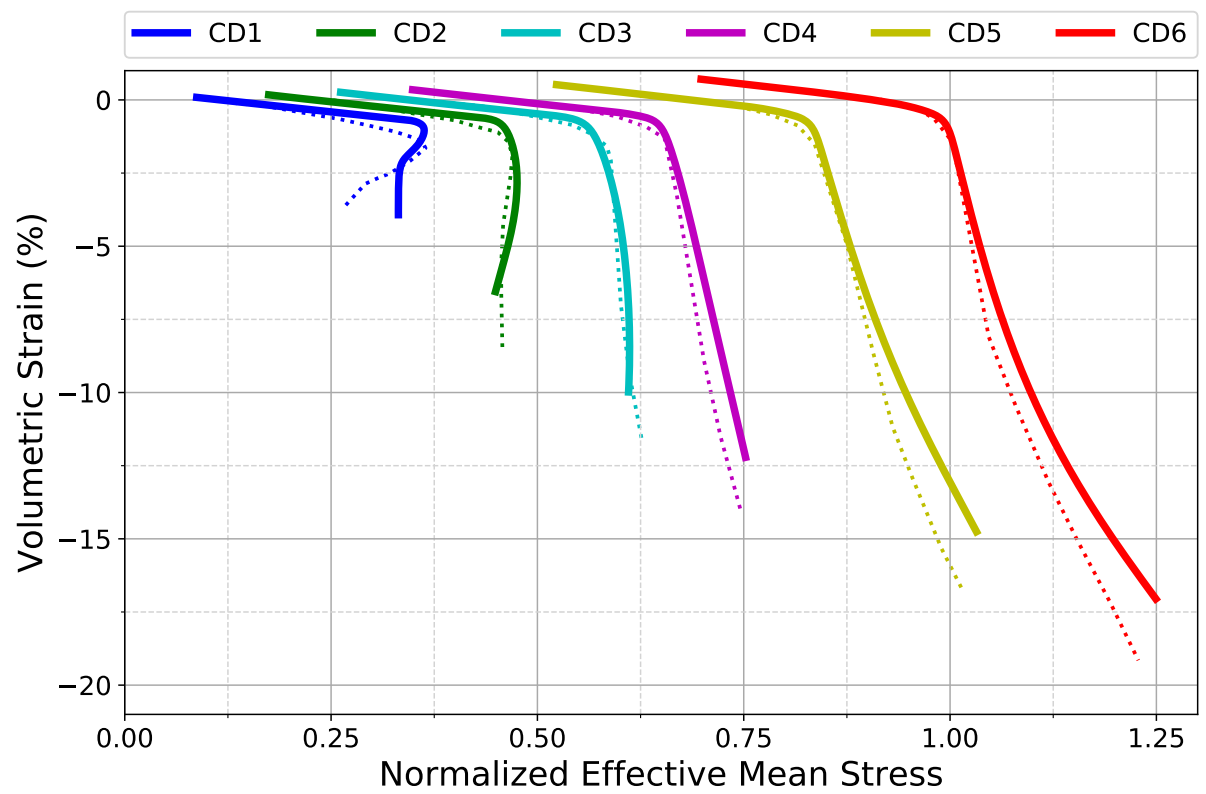

Figure 7: Volumetric strain curves in function of mean effective stress at various confinements for mudstone; comparison of numerical (solid lines) and experimental results (dashed lines). 


\subsection{Adamswiller sandstone}

In this second case study we use the inversion workflow of Sec. 3 to fit experimental data from triaxial tests performed on sandstone (Wong et al., 1997), a much harder material, to show that the workflow can operate in a wide range of conditions. This rock is composed of $71 \%$ quartz, $11 \%$ clay, $9 \%$ feldspar and $5 \%$ oxides and mica. Cylindrical samples of $18.4 \mathrm{~mm}$ diameter and $38.1 \mathrm{~mm}$ length, from cores parrallel to bedding, were subject to confined drained triaxial experiments at six different confining pressures of 5, 20, 40, 60, 100 and $150 \mathrm{MPa}$, named CD1-6. All samples were fully saturated with water and compressed up to $15 \%$ strain at a constant strain rate of $4 \times 10^{-5} s^{-1}$, after initialisation (see Wong et al., 1997, for details).

The inversion process followed the same steps described previously (see Sec. 4.1) and led to the identification of the yield enveloped presented in Fig. 8, using this time the preconsolidation value as a second free parameter. The numerical simulations show a good fit with experimental results, both for the deviatoric and volumetric responses, as shown in Fig. 9 and Fig. 10 respectively, for the corresponding parameter values listed in Table 4.2.

\begin{tabular}{|c|c|c|c|}
\hline Parameter name & Symbol & Value & $\begin{array}{l}\text { Unit of } \\
\text { Measure }\end{array}$ \\
\hline Flow law overstress exponent & $M$ & 2 & - \\
\hline Initial permeability & $\kappa$ & $\begin{array}{l}1.48 \\
10^{-14}\end{array}$ & $m^{2}$ \\
\hline Initial porosity & $\phi$ & 0.226 & - \\
\hline Arrhenius coefficient & $A r$ & 6 & - \\
\hline Young's modulus & $E$ & 9.45 & GPa \\
\hline Poisson ratio & $\nu$ & 0.2 & - \\
\hline Fluid thermal diffusivity & $c_{t h, f}$ & $\begin{array}{ll}1.43 & \times \\
10^{-7} & \end{array}$ & $m^{2} \cdot s^{-1}$ \\
\hline $\begin{array}{l}\text { Fluid thermal expansion coeffi- } \\
\text { cient }\end{array}$ & $\lambda_{f}$ & $6.9 \times 10^{-5}$ & $K^{-1}$ \\
\hline Fluid viscosity & $\mu, f$ & $8.9 \times 10^{-4}$ & Pa.s \\
\hline Fluid compressibility & $\beta_{f}$ & $\begin{array}{l}4.5 \\
10^{-10}\end{array}$ & $P a^{-1}$ \\
\hline Solid thermal diffusivity & $c_{t h, s}$ & $\begin{array}{l}1.64 \\
10^{-6}\end{array}$ & $m^{2} \cdot s^{-1}$ \\
\hline Soild thermal expansion coefficient & $\lambda_{s}$ & $3 \times 10^{-5}$ & $K^{-1}$ \\
\hline
\end{tabular}




\begin{tabular}{|l|l|l|l|}
\hline Solid compressibility & $\beta_{m}$ & $\begin{array}{l}1.9 \\
10^{-10}\end{array}$ & $\mathrm{~Pa}^{-1}$ \\
\hline Mixture thermal diffusivity & $c_{t h, m}$ & $1.3 \times 10^{-6}$ & $\mathrm{~m}^{2} \cdot \mathrm{s}^{-1}$ \\
\hline $\begin{array}{l}\text { Mixture thermal expansion coeffi- } \\
\text { cient }\end{array}$ & $\lambda_{s}$ & $3.9 \times 10^{-5}$ & $\mathrm{~K}^{-1}$ \\
\hline Mixture compressibility & $\beta_{m}$ & $\begin{array}{l}2.5 \\
10^{-10}\end{array}$ & $\mathrm{~Pa}^{-1}$ \\
\hline $\begin{array}{l}\text { Mixture thermal pressurisation co- } \\
\text { efficient }\end{array}$ & $\Lambda_{m}$ & $1 \times 10^{-6}$ & - \\
\hline $\begin{array}{l}\text { Lewis number } \\
\text { Gruntfest number }\end{array}$ & $\mathrm{Le}$ & 0.65 & - \\
\hline $\begin{array}{l}\text { Yield envelope, tension cut-off } \\
\text { Yield envelope, preconsolidation } \\
\text { pressure }\end{array}$ & $p_{t}$ & 0.3 & - \\
\hline $\begin{array}{l}\text { Yield envelope, slope of critical } \\
\text { state line }\end{array}$ & $M$ & -5 & $\mathrm{MPa}$ \\
\hline $\begin{array}{l}\text { Yield envelope, } \alpha \text { parameter } \\
\text { Yield envelope, } \beta \text { parameter }\end{array}$ & $\alpha$ & 6 & $\mathrm{MPa}$ \\
\hline
\end{tabular}

Table 4: Parameter values obtained for the fits of sandstone experiments shown in Fig. 9 and Fig. 10.

\subsection{Inversion of mechanical enthalpy}

Experience showed that the calibration of the parameters $\alpha_{1}$ and $\alpha_{2}$ of the mechanical enthalpy proved rather difficult, which triggered the need for a specific sensitivity analysis of those parameters.

\subsubsection{Sensitivity analysis}

Keeping all other parameters constant, a parameter study was then performed on $\alpha_{1}$ and $\alpha_{2}$ (from Eq. 6) by taking 25 equally spaced values of those parameters within given ranges $\left(\alpha_{1}\right.$ in $[-8.5,-2.5], \alpha_{2}$ in $\left.[0,60]\right)$ and running every other cross-product simulations to cover that parameter space. The 313 simulation results produced were subsequently compared against the experimental data using the objective function described in Sec. 3.3 to produce sensitivity maps, shown on the top row of Fig. 11 for the cases CD4-6 of mudstone. These maps highlight the existence, for each separate case, of a zone in $\left(\alpha_{1}, \alpha_{2}\right)$ producing relatively good fits (low objective function values) 


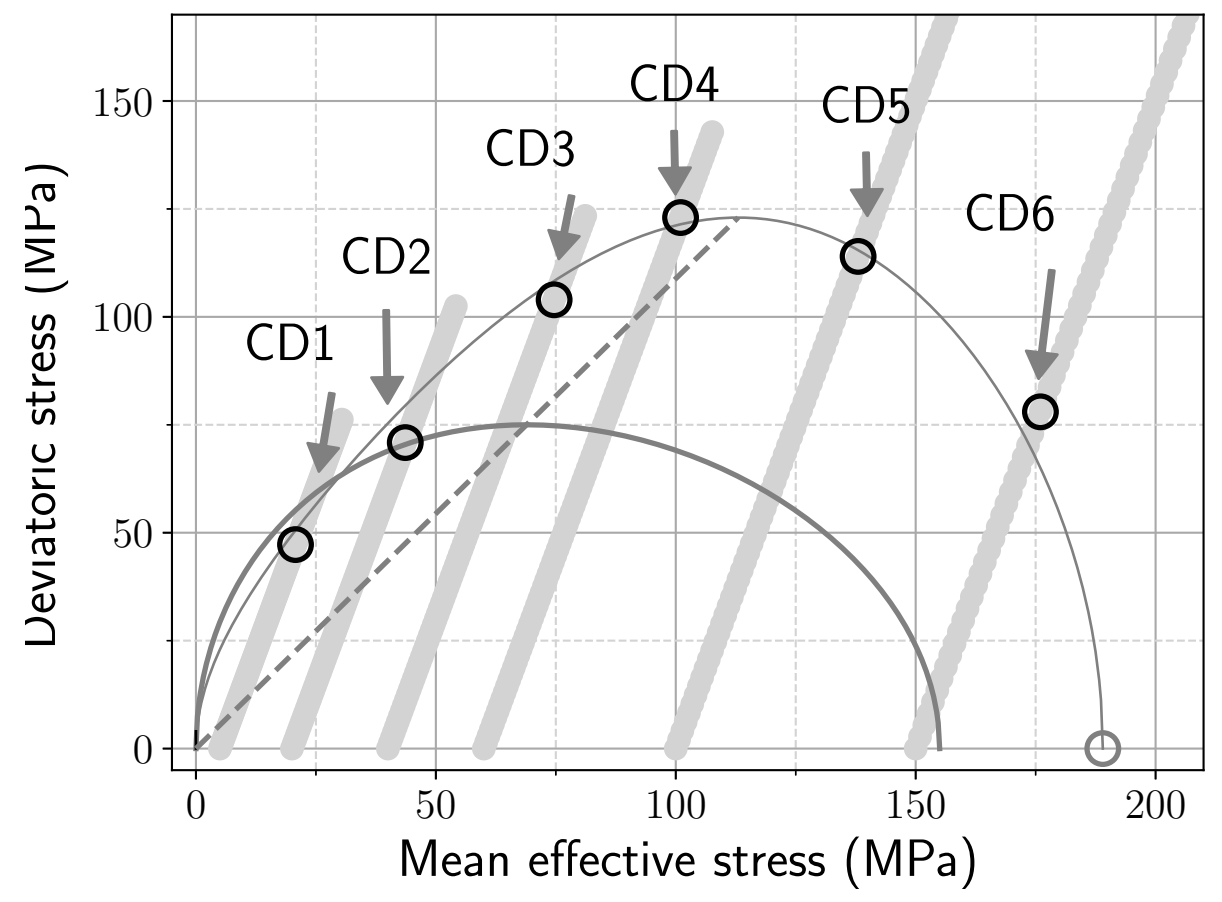

Figure 8: Experimentally determined yield points (black circles) for sandstone by Wong et al. (1997) and corresponding yield envelope (thick line), as well as result yield envelope (think line) obtained from the inversion process, scaling the experimental yield envelope along the Critical State Line (dashed line) with a shift in pre-consolidation pressure. The thick lines in light grey show the stress paths for all confined drained experiments CD1-6. 


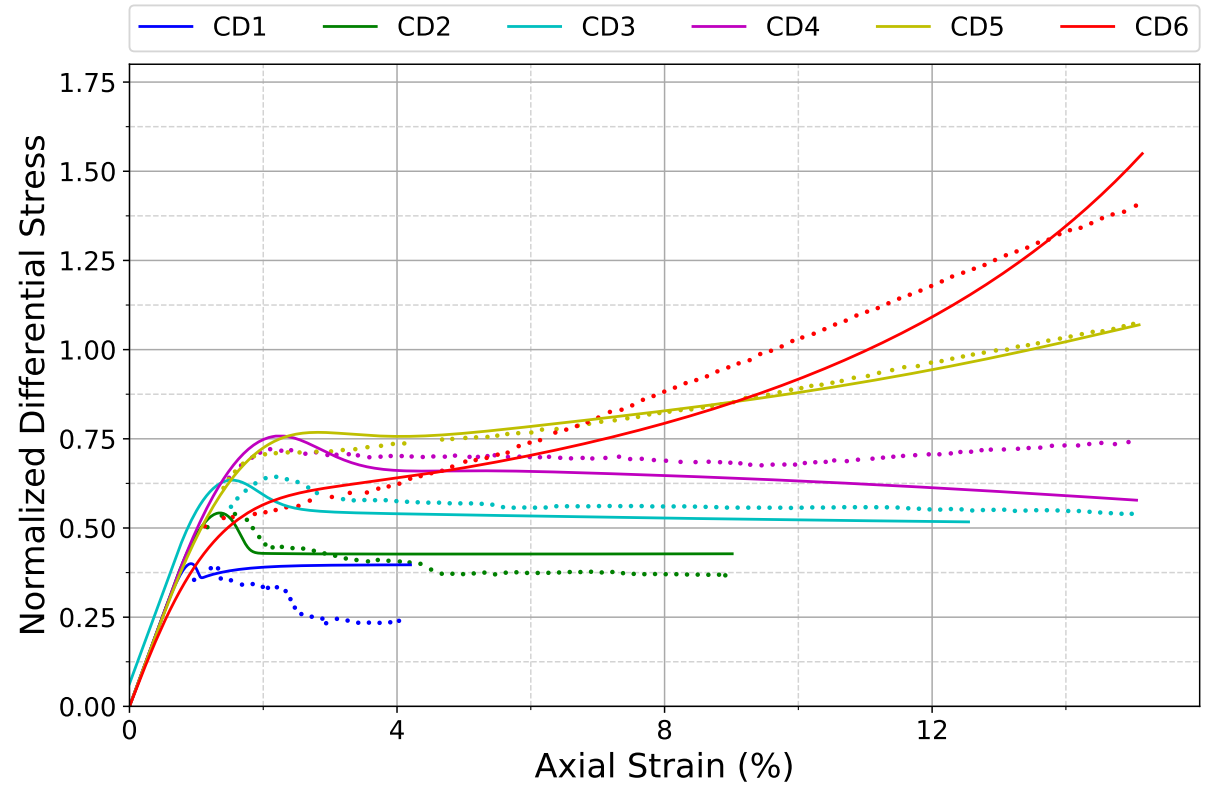

Figure 9: Stress strain curves at various confinements for sandstone; comparison of numerical (solid lines) and experimental results (dashed lines). 


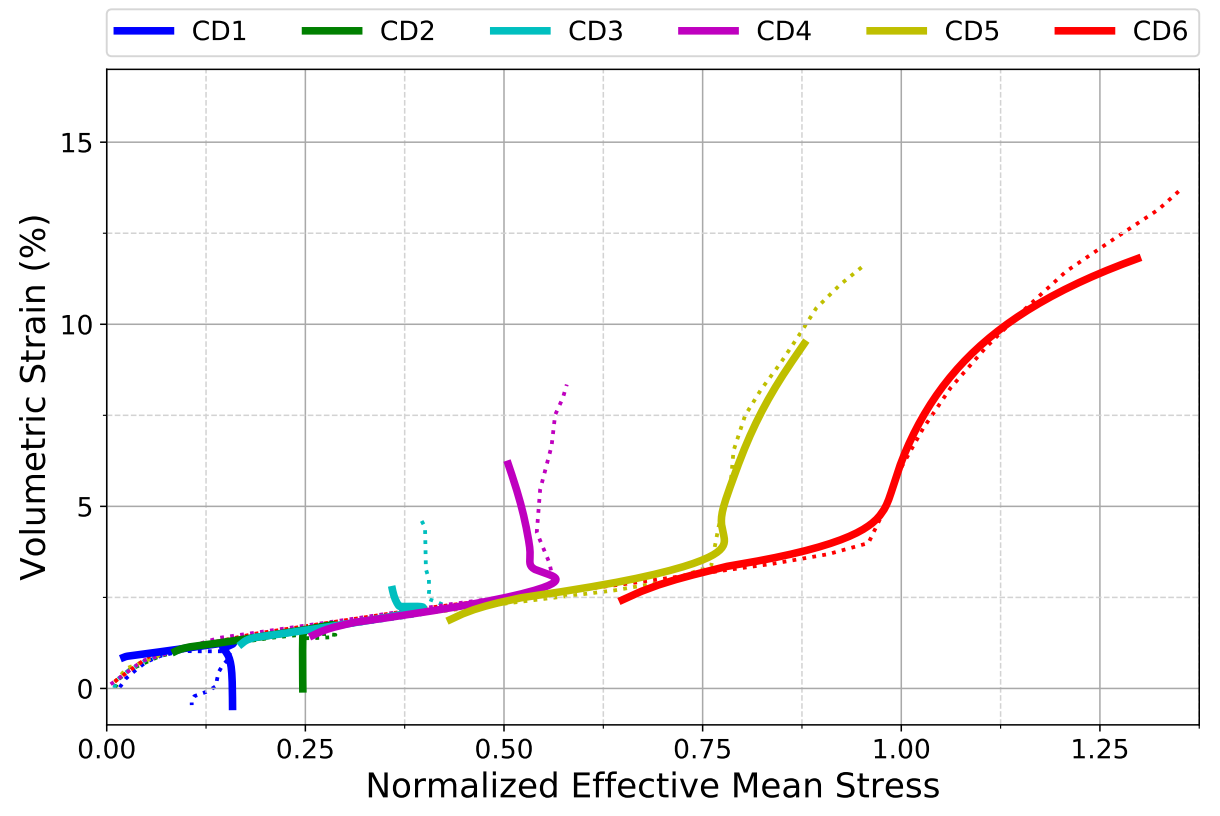

Figure 10: Volumetric strain curves at various confinements for sandstone; comparison of numerical (solid lines) and experimental results (dashed lines). 
CD4

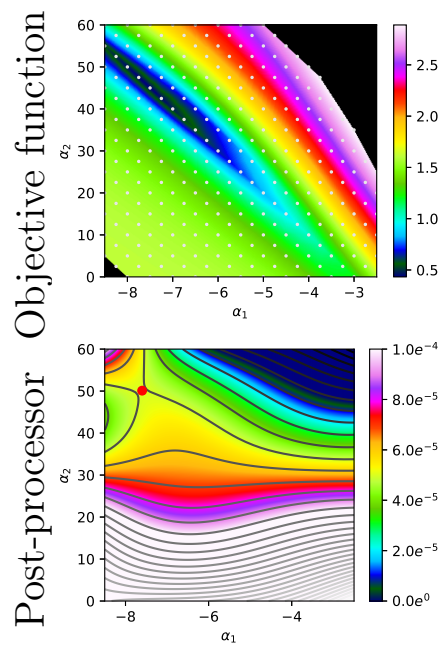

CD5
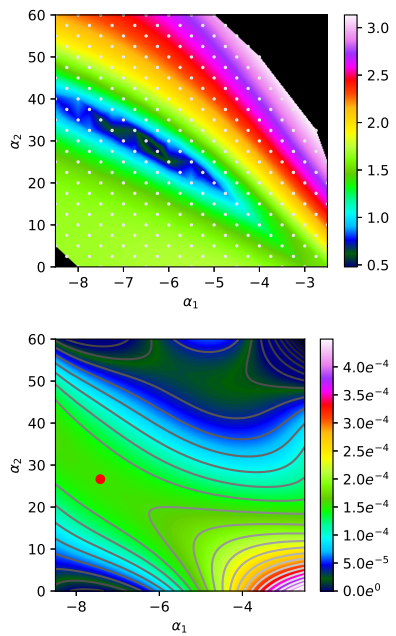

CD6
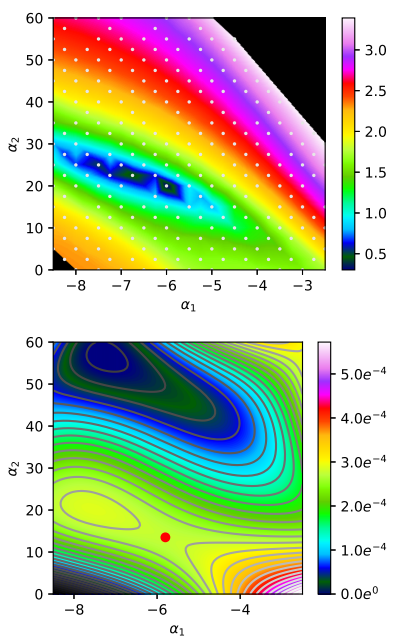

Figure 11: Sensitivity analysis in $\left(\alpha_{1}, \alpha_{2}\right)$ parameter space for mudstone experiments CD46. The top row displays the value of the objective function interpolated from 313 simulations (white dots), showing a localised zone of good fits (low values). The bottom row shows the corresponding values of a postprocessor computing the volumetric component of the plastic mechanical work at the center of the sample. The red dot shows the location of identified saddle points. Note that some colorbars were capped to exclude extreme values and better illustrate the patterns.

and potentially hosting a single global minimum, even though it can not be precisely located with the coarse sampling resolution used.

A simple optimisation process could easily locate the global minimum precisely in a much reduced number of simulations than the 313 run ones. The advantage of this heurstic visualisation of the full map, however, is to provide an important understanding to theoretical modellers about the behaviour of the specific model selected (see Sec. 2). An underlying structure appears indeed quite clearly on those maps, which would not be so obvious through a blind optimisation approach. Even more interestingly, these maps allow us to proceed with various numerical inquiries to try and investigate whether the pattern observed on the sensitivity maps of the objective function in the $\left(\alpha_{1}, \alpha_{2}\right)$ parameter space could be identified from the numerical results only, therefore reducing strongly the amount of laboratory experiments required. 


\subsubsection{Saddle point in volumetric plastic work}

The volumetric component of the plastic mechanical work is of particular interest, computed at the center of the (virtual) rock sample as a postprocessor for all 313 simulations, using the same parameter sampling in the $\left(\alpha_{1}, \alpha_{2}\right)$ space as described in the previous section. The results of this postprocessor for each simulation are then interpolated using a polynomial fit of order 3 in both directions $\left(\sum_{i, j=0}^{3} a_{i j}\left(\alpha_{1}\right)^{i}\left(\alpha_{2}\right)^{j}=0\right)$, which provides a very accurate fit that is now smooth enough to compute precisely the location of characteristic points of this function of two variables.

The bottom row of Fig. 11 shows that all maps display patterns that are reflecting the ones observed in the objective function maps, along with the presence of a saddle point, which can be computed accurately on the polynomial approximation.

\section{Discussion and conclusion}

This study showcased a heuristic inversion workflow for calibrating a thermo-hydro-mechanical model based on elasto-visco-plasticity against triaxial experimental data. We demonstrated the ability of the underlying physical model to capture both the volumetric and deviatoric behaviours of rock deformation for two different cases, mudstone (see Sec. 4.1) and sandstone (see Sec. 4.2), building on previous work which had showed promising results on mudstone for the deviatoric behaviour only (Poulet and Veveakis, 2016). The heuristic nature of the approach is an important component, both from theoretical and numerical reasons. Numerically, the multi-physical nature of the model obviously grows considerably the number of parameters that need to be inverted, which comes at an exponential computational cost in terms of simulations needed to parse the parameter space. As such, the breakdown of the inversion process in three separate stages, as described on Fig. 3, provides individual steps that are much more manageable computationally. The numerical argument, however, does not represent the major reason for this breakdown, which is even more important for theoretical reasons. Those stages represent indeed a much better way for theoretical modellers to understand the behaviour of their physical model in terms of parameter sensitivity, compared with a single-step optimisation approach. In practise, the data driven approach presented here provided a better understanding of the flow law description used (Eq. 5) and led to the discovery of a potentially 
game-changing property of the model with the presence of a saddle point (see Sec. 4.3.2).

The time and cost of running laboratory experiments are important factors to take into consideration when characterising a rock behaviour and using numerical simulations to minimise those costs is certainly very appealing. The potential presence of a saddle point in the volumetric component of the plastic mechanical power, as shown in Sec. 4.3.2, is therefore a critical outcome of this study as it could help reduce the number of laboratory experiments needed to calibrate this physical model. The theoretical study of the physical reasons for this behaviour clearly falls outside the scope of this work, but it is important to note the role of a data-driven analysis to infer information about a theoretical model to help its development. This reinforces the significance of taking a heuristic approach and highlights the need not to de-correlate too quickly the complementary physically-driven and data-driven approaches, as they both strongly benefit from a tight and simultaneous interaction.

It is also worth mentioning some other conclusions drawn from this calibration exercise regarding the concept of the yield envelope, as well as the important physical roles of temperature, pressure and rate sensitivity of materials.

The distinction between an experimentally obtained yield envelope at the sample scale and the yield surface required at the smaller scale for equivalent finite element simulations has always been an admitted concept in theory. In this contribution, we considered the possibility to use a scaled-down version of the experimental curves (see Sec. 2.4.2) and showed satisfactory modelling results which justify further investigations of this approach. Once again, the theoretical justification of this method falls outside the scope of this study but we highlight the important role of numerical investigations to help infer theoretical knowledge on constitutive relationships.

The results obtained in Sec. 4 demonstrate as well the temperature, pressure and rate dependency of the materials tested, showing that it is possible to capture relatively well already the physical reasons behind the puzzling hardening/weakening evolution with confining pressure. While Sec. 2 presented theoretical reasons to take into account those mechanisms, the interpretation of the results justifies those considerations.

Most models treat triaxial experiments as isothermal, which is not necessarily justified a priori as discussed in Sec. 2.1. From the results obtained we can now illustrate more precisely effect of considering temperature varia- 

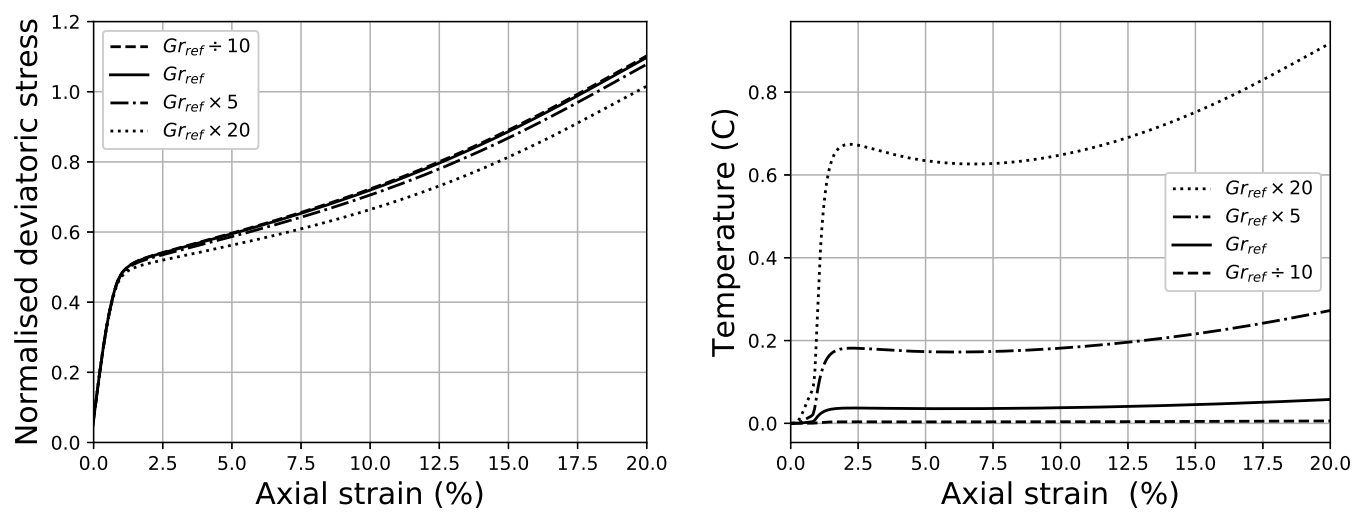

Figure 12: Sensitivity analysis on Gruntfest number $G r$, showing noticeable temperature weakening on the stress-strain curves (left) for corresponding temperature values (right) not exceeding $1^{\circ} \mathrm{C}$ at the centre of the 3D block.

tions, at least in terms of overall impact since its effects are feeding back on pore pressure and stress through the various feedbacks considered in the system of equations (5a-c). Fig. 12 shows the thermal weakening of the model and the sensitivity of the Gruntfest number $G r$, encompassing the microstructural shear heating effects, on the stress-strain response of mudstone at $1.5 \mathrm{MPa}$ confinement for instance. We can observe a slight weakening when increasing $G r$ by a factor 5, while the maximum temperature changes (at the centre of the virtual rock sample) remain below a maximum of $0.3^{\circ} \mathrm{C}$. These minor temperature variations within the sample would remain barely noticeable from the outside using a recent thermal infrared camera, which shows the important effects of even minor temperature changes. Note as well the temperature feedback on the mechanical behaviour through the temperature dependency of the mechanical flow law and the Arrhenius parameter $\operatorname{Ar}$ (Eq. 5), with a sensitivity analysis for the same simulation shown in Fig. 13. The effects on the mechanical response are even more drastic, with temperature changes yet remaining once again below noticeable values from the outside.

Similarly to temperature, pore pressure effects also play an important role in the results obtained and highlight the range of values required for the Lewis number Le for the pressure sensitivity of the flow law to become relevant. While the definition of Le from Eq. (4a) only accounts for Darcy flow in terms of mass diffusivity, the higher values obtained through the inversion - 

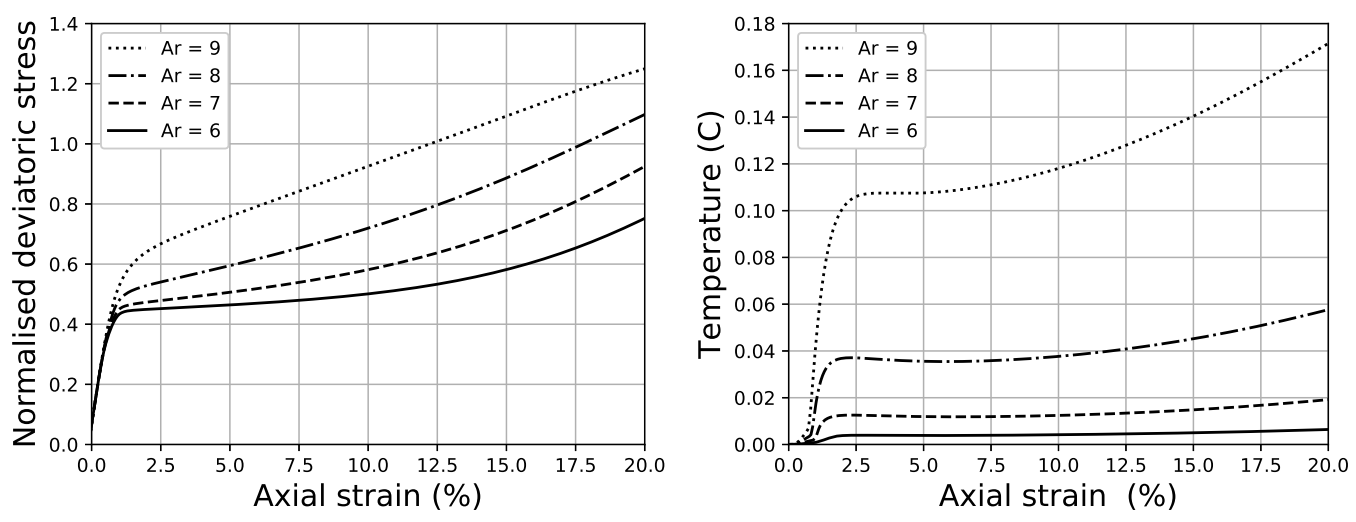

Figure 13: Sensitivity analysis on Arrhenius number $A r$, showing extremely different mechanical responses on the stress-strain curves (left) for corresponding temperature values (right) not exceeding $0.2^{\circ} \mathrm{C}$ at the centre of the $3 \mathrm{D}$ block.

up to 5 orders of magnitude for mudstone for instance point to the fact that other mechanisms are at play and should be accounted for in the physical model. This finding highlights once again the importance of the numerical approach to help derive the physical model, and to allow flexible bounds for poorly constrained numbers (like $L e$ ) as a good way to infer new directions for the theoretical model development. For instance, Sari (2019) proposed recently a new formulation of the mechanical flow law accounting for the mass diffusivity of a weaker solid phase resulting from grain debonding and cement breakage, which would indeed translate in our model as much larger values of $L e$.

Finally, the rate sensitivity built in the model has already been demonstrated (Poulet and Veveakis, 2016) and proved as well to play a role in the calibration studies presented here. Considering creep brings more focus on the impact of the loading rates used to equilibrate the laboratory experiments at high confinements, especially as we considered reduced yield envelopes. This raises the interesting question whether some creep had actually started in places, at the microstructural level, for the experiments at highest confinements. This question can only be answered in the laboratory but points again at the over-reaching impact of the data-driven numerical approach beyond its originally intended scope.

In conclusion, this study demonstrated the importance of linking theoretical and numerical investigations as both approaches are so complementary 
in the context of multiphysics that they cannot satisfactorily be tackled independently. The workflow presented here provides a practical step towards this goal and showed the impact of combining physically-driven and datadriven approaches. The formulation of a physical model accounting directly for temperature and pressure evolution could open the door to possible extrapolations of existing laboratory results beyond the conditions previously tested (high temperature, high pressure, goeological strain rates) and such novel theories will certainly require an even tighter experimental-theoreticalnumerical integration.

\section{Acknowledgements}

The authors acknowledge support by the Australian Research Council (ARC Discovery grants no. DP170104550, DP170104557), the DE-NE0008746DoE project and UNSW Sydney. This research was undertaken with the assistance of resources and services from the National Computational Infrastructure (NCI), which is supported by the Australian Government. This work was also supported by resources provided by the Pawsey Supercomputing Centre with funding from the Australian Government and the Government of Western Australia.

\section{References}

Gerolymatou, E., 2017. Induced and inherent anisotropy in rock mass. Ph.D. thesis, Fakultät für Bauingenieur-, Geo- und Umweltwissenschaften (BGU), Karlsruher Institut für Technologie, habilitation.

Lin, J., Sari, M., Poulet, T., Veveakis, M., 2017. An Inversion Framework for Numerical Modelling of Pore Collapse in Soft Porous Rocks. Springer International Publishing, Cham, pp. 319-325.

Mielke, A., 2006. Multifield Problems in Solid and Fluid Mechanics. Lecture Notes in Applied and Computational Mechanics. Springer Berlin / Heidelberg, Ch. A Mathematical Framework for Generalized Standard Materials in the Rate-Independent Case, pp. 399-428.

Nguyen-Tuan, L., Lahmer, T., Datcheva, M., Stoimenova, E., Schanz, T., 2016. A novel parameter identification approach for buffer elements involving complex coupled thermo-hydro-mechanical analyses. Computers and Geotechnics 76, $23-32$. 
Oka, F., Kimoto, S., Higo, Y., Ohta, H., Sanagawa, T., Kodaka, T., 2011. An elasto-viscoplastic model for diatomaceous mudstone and numerical simulation of compaction bands. International Journal for Numerical and Analytical Methods in Geomechanics 35 (2), 244-263.

Perzyna, P., 1966. Fundamental problems in viscoplasticity. Advances in Applied Mechanics 9, $244-377$.

Poulet, T., Veveakis, M., 2016. A viscoplastic approach for pore collapse in saturated soft rocks using redback: An open-source parallel simulator for rock mechanics with dissipative feedbacks. Computers and Geotechnics 74, $211-221$.

Sari, M., 2019. A multi-scale multi-physics plasticity theory for porous sedimentary rocks. Ph.D. thesis, UNSW Minerals and Energy Resources Engineering, Sydney, Australia, (under review).

Wijns, C., Poulet, T., Boschetti, F., Dyt, C., Griffiths, C., 2004. Interactive inverse methodology applied to stratigraphic forward modelling. Geological Society Special Publication 239, 147-156.

Wolpert, D. H., Macready, W. G., April 1997. No free lunch theorems for optimization. IEEE Transactions on Evolutionary Computation 1 (1), 6782.

Wong, T.-f., David, C., Zhu, W., 1997. The transition from brittle faulting to cataclastic flow in porous sandstones: Mechanical deformation. Journal of Geophysical Research: Solid Earth 102 (B2), 3009-3025.

Wu, L., Liu, S., Wu, Y., Wang, C., 2006. Precursors for rock fracturing and failure part i: Irr image abnormalities. International Journal of Rock Mechanics and Mining Sciences 43 (3), 473 - 482. 